\title{
The Speckled Golfer
}

\author{
D. K. Arvind \\ Research Consortium in Speckled Computing \\ School of Informatics \\ University of Edinburgh \\ Mayfield Road, Edinburgh EH93JZ \\ Scotland, UK \\ +441316505176 \\ dka@inf.ed.ac.uk
}

\author{
Andrew Bates \\ Research Consortium in Speckled Computing \\ School of Informatics \\ University of Edinburgh \\ Mayfield Road, Edinburgh EH93JZ \\ Scotland, UK. \\ $+447825564480$
}

Andrew.bates11@gmail.com

\begin{abstract}
This paper describes the application of the first fully wireless network of inertial sensors for full-body 3-D motion capture - the Orient-2 system, to real-time on-body analysis of the golf swing. The golfer-club system is modelled as a double pendulum. The factors which affect the efficiency of the golf swing are outlined, such as the length of the back swing, the wrist-cock angle, swing plane and club-head speed and methods are described for monitoring them using a wireless network of Orient-2 specks. The implementation of the Speckled Golfer System is described and two motion rules are illustrated for executing efficient golf swings.
\end{abstract}

\section{Keywords}

Body Sensor Networks, Wireless Sensor Networks, Wireless Motion Capture, Golf Swing Analysis.

\section{INTRODUCTION}

The application of physics to the analysis of the golf swing has been the subject of a number of studies [1][2][3][4]. The golf swing is modelled as a double pendulum; the arms and the hands of the golfer act as the upper pendulum swinging about the fixed pivot of the shoulders, and attached to the free end of this pendulum is the club shaft and club head (the lower pendulum). At the bottom of the swing of the club the kinetic energy is transferred from the club-head to the ball on impact, and faster the club-head, greater is the momentum imparted to the ball and greater is the velocity at which it takes off from a stationary start. A good swing technique differentiates the expert golfer from the novice in being able to transfer energy efficiently from the clubhead to the ball. The swing involves a series of complex interactions between different parts of the golfer's body and accompanied with shifting of body weight from one leg to the other. At the start of the swing, the golfer reaches far back while

Permission to make digital or hard copies of all or part of this work for personal or classroom use is granted without fee provided that copies are not made or distributed for profit or commercial advantage and that copies bear this notice and the full citation on the first page. To copy otherwise, to republish, to post on servers or to redistribute to lists, requires prior specific permission and/or a fee.

BodyNets 2008, March 13-15 Tempe, Arizona, USA

Copyright (C) 2008 ICST 978-963-9799-17-2

DOI 10.4108/ICST.BODYNETS2008.2956 the torso rotates above the right leg. As the swing begins, the golfer builds up club-head velocity by rapidly turning the torso and driving through the ball using his hips, shoulders and wrists to transfer the momentum from the club (the impact lasts about 0.5 millisecond), while maintaining the position of the head above the ball. The momentum of the club extends a smooth follow through around the back. The key observation is the need to co-ordinate the entire body during execution of a golf swing, and the aim of this paper is to demonstrate the viability of using an on-body network of Orient-2 [5] inertial sensor specks [6] to track and analyse the complex interactions between different parts of the body. The result of the analysis is a measure of the quality of the swing in terms of how closely it obeys a set of motion rules which is fed back in real-time to the golfer.

The Orient-2 inertial sensor speck and wireless network [5] developed by the Research Consortium in Speckled Computing [7] at the University of Edinburgh, demonstrated for the first time, fully wireless, full-body 3-D posture capture in real-time using a network of 15 unobtrusive (36mmX28mmX11mm) devices, at an update rate of $64 \mathrm{~Hz}$ over the wireless network and a latency of 20 milliseconds, using a modest low-power $250 \mathrm{kbps}$ radio. The key innovation was an efficient algorithm for localised orientation estimation, which has resulted in significant reduction (79\%) in communication data compared to existing methods. This work has freed motion capture from the confines of a camera-equipped studio, which has excited end-users in diverse applications ranging from biomechanics (gait analysis, physiotherapy, sports), animation, and robotics.

This paper describes the implementation of a body-based wireless sensor network for capturing the motion of a golf swing and providing real-time sonic feedback or visual feedback on a PC or a PDA. A number of motion rules are described which are weighted according to their importance and combined to provide an overall score.

The approach described in this paper can be generalised to any sequence of movements governed by a set of rules for their correct execution, such as in gymnastics, dance, or martial arts. 


\section{CAPTURING THE GOLF SWING}

Traditional methods for analysing golf swings have used stroboscopic photography or video motion capture to obtain space-time data in the studio.

\subsection{Stroboscopic Photography}

One of the earliest methods [1] for analysing golf swing employed stroboscopic photography to capture the positions of the club and parts of the body of the golfer which were highlighted with reflective tapes.

These studies used a normal exposure for the golfer and produced a blurred photograph consisting of many superimposed images of the swing. Shooting in near darkness with an exposure suited to the reflective tape produced a clearer photograph composed of dots showing positions at different time instants at each flash of the strobe.

A flash rate of $120 \mathrm{~Hz}$ gave approximately 150 dots on the photograph from the start of the backswing to when the ball was hit. The positions and distances between the dots could then be measured to determine the orientation and speed of the club or parts of the golfer during the swing. Capturing too much information about the swing in a single photograph produced a very busy negative which was difficult to interpret. Therefore several photographs were taken with the reflective tape in slightly different positions.

\subsection{Video camera-based motion capture}

Video camera-based motion capture has been used extensively to capture the golf swing within a studio environment, which also requires significant post-processing time to generate orientation data from the video footage. Although video cameras could be set up on the golf course, it would be inconvenient during a real game of golf. Occlusion can be a problem unless multiple cameras are used. Many systems are limited to 30 frames-per-second, which is significantly slower than most wireless sensor-based motion capture systems, which can achieve hundreds of updates per second.

In summary, the camera-based motion capture methods described previously are not well suited for capturing the motion of a golfer's swing, analysing it and providing feedback in real-time on the golf course. Both methods require significant postprocessing to determine the orientation and speed of the club at a particular point during the swing. This makes it impossible to provide real-time feedback to the golfer immediately after a swing. In addition, these methods require careful positioning of the cameras to avoid occlusion and the presence of a controlled light source.

\subsection{Wired inertial sensor-based system}

An inertial wireless sensor network can be used to directly determine the orientation of the golf club and the limbs of the golfer to provide real-time feedback to the golfer on the course. Full-body motion capture suits such as Moven from Xsens [8] can track the orientation of the golfer's limbs, but not the combination of the golfer and the club. This is because the sensor devices are connected by wires and attached to a lycra suit worn by the golfer and synchronisation with an external sensor device on the golf club has to be engineered separately. The wired sensor network is able to communicate wirelessly with the base-station.

\section{WIRELESS INERTIAL SENSOR-BASED SYSTEMS}

\subsection{Sonic Golf}

The Sonic Golf system consists of a single wireless sensor that is inserted into the shaft of the club [9], which measures the speed of movement of the club. A base-station worn on a belt converts the sensor measurements into an audio tone that is transmitted to a pair of wireless headphones to provide real-time feedback to the user.

Sonic Golf neither performs full motion tracking of the club, nor does it sense the movement or orientation of parts of a golfer's body.

\subsection{Smart Swing}

Smart Swing tracks the motion of the golf club, using a sensor containing a gyro, accelerometer and magnetometer for each axis [10]. The sensor communicates wirelessly to a base station, which is connected to a laptop. An animation of the swing is shown and a score is produced, based on how closely the swing matches a reference swing.

Smart Swing does not provide instantaneous feedback to the user and no audio feedback is available. The system is limited to a single device in the club and does not support a network of sensors on the golfer.

The device is approximately $15 \mathrm{~cm}$ long and fits in a golf club with an inside diameter of $12 \mathrm{~mm}$. It provides an update rate of $1000 \mathrm{~Hz}$, which is significantly higher than the other systems discussed here.

\subsection{K-Vest}

The K-Vest [11] provides two wireless sensors in a vest; one on the hip and one on the shoulder. A third sensor is worn on the back of the hand, which is used to track the motion of the golf club.

The sensor used is the InterSense IntertiaCube [12] which combines 3-axes magnetometer, gyro and accelerometer with a radio. These sensors offload raw sensor data to the PC for processing. The K-Vest system is limited to a maximum of four devices for an update rate of $120 \mathrm{~Hz}$.

The sensor cannot be placed on the club, given its large size and weight of approximately 50 grams, which was found to affect the feel of the club. However, satisfactory results have been obtained by placing the sensor on the hand rather than on the club.

The system provides a real-time audio and visual feedback to the golfer and can display joint angles. It also provides a 3-D animation of the golfer, which can be coloured to highlight faults in the golf swing.

3.4 Orient-2 Wireless Motion Capture System The Orient-2 hardware [5] developed by the Research Consortium in Speckled Computing [7] at the University of Edinburgh 
consists of four interlocking PCBs assembled to form a 3-D structure measuring 36 X 28 X 11mm which surrounds a 120 mAh lithium polymer battery and weighs 13 gms. The processor is a Microchip dsPIC 30F3014 16-bit microcontroller which is clocked from a 7.37 internal RC oscillator and a $32,768 \mathrm{KHz}$ crystal oscillator provides a real-time clock. The processor has $2 \mathrm{~KB}$ of SRAM and $24 \mathrm{~KB}$ of program memory, together with $1 \mathrm{~KB}$ of onboard EEPROM. A 4MB external Flash memory chip is included for data logging. A Chipcon CC1100 transceiver is used for radio communications which operates at $868 \mathrm{MHz}$ ISM band at a data rate of $250 \mathrm{Kbps}$. The sensors used are a Freescale MMA7260Q three-axes accelerometer, two Honeywell HMC1052 two-axes magnetometers, and three Analog Devices ADXRS300 MEMS rate gyroscopes.

The firmware on the processor carries out all aspects of real-time operations, including sampling and normalisation of sensor data and orientation estimation, and communications such as the transmission and handling of data and control packets.

\section{PHYSICAL MODEL}

The arms of the golfer and the club are modelled as two rigid rods, connected at the wrist [1]. The arms and the club act as a double pendulum. The first pendulum pivots about the shoulder of the golfer and the club acts as a second pendulum, pivoting about the golfer's wrists.

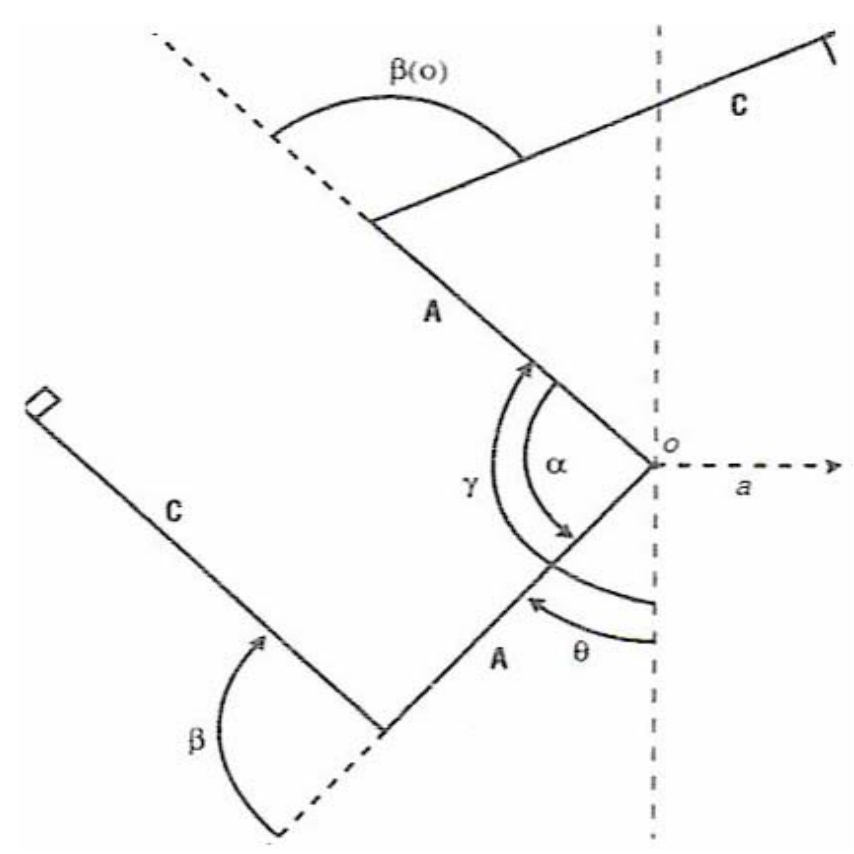

Figure 1: Physical model of the golf swing[1], where:

- A: The golfer's arm

- C: The club

- a: horizontal acceleration at $\mathrm{O}$

- $\gamma$ : angle of $\operatorname{rod} A$ with the downward direction at the start of the downswing

- $\quad \beta$ : wrist-cock angle

- $\quad \theta$ : downward angle of $\operatorname{rod} A$

- $\alpha$ : downward swing angle
By placing the Orient-2 devices on the club, the forearm, the upper arm and the back of the golfer, the angles shown in Figure 1 can be measured. The value of these angles at different stages of the swing can be important for determining the speed of the club during the swing and the momentum imparted to the ball on impact.

The Orient-2 device on the back acts as the root node of the body model, so that the orientation of the other joints will be relative to this device. This implies that the golfer can move around without changing the orientation of the body model joints.

The factors which affect the efficiency of the golf swing include the length of the player's arms, the mass and length of the club, the mass of the club-head, the wrist-cock angle and the wrist torque.

\subsection{The Length of the Backswing}

The length of the backswing is shown by angle $\gamma$ in Figure 1 . Although a large backswing can produce a powerful swing, it also reduces the accuracy of the shot. Many golfers will improve their swing by reducing the backswing angle. The backswing angle can be determined by the orientation of the Orient- 2 mounted on the golfer's upper arm.

\subsection{Wrist-cock angle}

The wrist-cock angle is how far backwards the wrists are bent during the swing, and the variation of the wrist-cock angle $(\boldsymbol{\beta})$ during the swing is important, because it is possible to exert a torque by actively twisting the wrist during the swing, called the wrist torque, that reduces the impact speed of the club with the ball. This can be measured by monitoring the difference in orientations between the Orient-2 device on the club and the forearm. The influence of the wrist-cock angle on the redistribution of energy between the arms and the club during the swing is described next.

\subsection{Energy transfer}

Much of the energy of the golf swing is provided by the large muscles of the body such as the legs and back. This energy is transmitted to the arms and club by accelerating the left shoulder (A) horizontally to move the pivot point of the arm (O). An Orient-2 speck mounted on the shoulder or hips of the golfer can measure this acceleration.

White's analysis [2] showed that the uncocking of the wrists that takes place before the club strikes the ball has a big impact on the efficiency of the swing. As the wrists uncock near the bottom of the swing, the club pulls against the hands and slows them down, and in turn the energy in the shoulders and arms is transferred to the club. These variations can be monitored by the Orient- 2 speck as mentioned before.

\subsection{The swing plane}

During the backswing and downswing phases, the club should be moved in a single plane that is tilted from the vertical. Any deviation from this plane can be detected by monitoring the angle between the plane and a vector pointing down the shaft of the 
club. A more detailed description for calculating deviation from the swing plane is given in Section 6.1.

\subsection{Club-head Speed}

The speed of the club-head as it hits the ball can be calculated from the rates of change of angles $\boldsymbol{\alpha}$ and $\boldsymbol{\beta}$. The point at which the ball is hit can either be detected by when the club is vertical, or by a spike in the acceleration data caused by the impact with the ball. Given the length of the golfer's arm and the length of the club, it is then possible to calculate the linear velocity of the club-head as it hits the ball.

\section{MOTION SCORING SYSTEM}

The orientation data from the Orient-2 devices is applied to a body model to give the relative orientation of the golfer's joints as described in Section 4.

The motion scoring system extracts snapshots of the orientation data from the body model throughout the golf swing. The orientation information from each snapshot is passed to each of the motion rules. This consists of a timestamp and an orientation quaternion for each joint in the body model. Acceleration data can also be provided if required by the motion rule.

Each motion rule produces a score, between zero and one, which is a measure of how well the current motion obeys the rule. Some motion rules only use data from the current snapshot, whereas others use historical data to measure how the orientation of a joint has changed over time.

The score for each rule is weighted according to its importance, and an overall score is calculated for the current snapshot of motion. The weighting of each rule can be set at run-time by the user, so one can choose to concentrate on improving a particular aspect of the motion of the club. Additional data regarding the motion can be made available to the user, such as an absolute angle reading that is important to a particular motion rule.

The scores and additional information from each rule, along with the overall score, are presented to the golfer in real-time during the swing, using graphical and audio feedback, as described in Section 7.

\section{MOTION RULES FOR GOLF SWING}

Two examples are presented of motion rules for a golf swing, and for each rule, calculations are shown that convert quaternion orientation data into a numerical score that can be used by the motion scoring system.

The normal to the swing plane, $\mathbf{n}$, is calculated by taking the cross product of $\mathbf{v}_{\text {start }}$ and $\mathbf{v}_{\text {end }}$, and is therefore perpendicular to both $\mathbf{v}_{\text {start }}$ and $\mathbf{v}_{\text {end }}$. As both these vectors must lie in the swing plane, $\mathbf{n}$ must be a normal to the plane.

\section{1 'Swinging in a Plane' Rule}

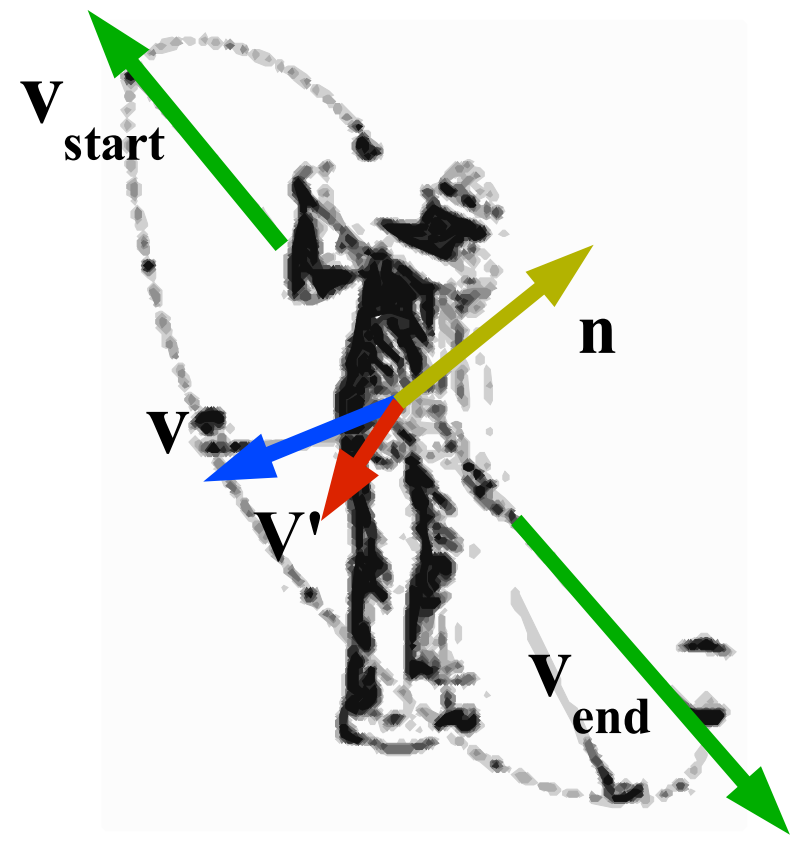

Figure 2: The Swing Plane, where:

- $\mathbf{v}_{\text {start }}$ : Vector pointing down the shaft of the club at the start of the swing

- $\quad v_{\text {end }}$ : Shaft vector at the end of the swing

- $\quad$ n: A normal to the swing plane, which contains both $\mathbf{v}_{\text {start }}$ and $\mathbf{v}_{\text {end }}$

- $\quad v^{\prime}$ : A general shaft vector during the swing, which does not lie in the plane

- v: The correct shaft vector, which is in the plane defined by $n$

The angle, $\boldsymbol{\alpha}$, between the shaft of the club and the ideal swing plane normal is the angle between $\mathbf{v}^{\prime}$ and $\mathbf{n}$, which is given by $\cos (\boldsymbol{\alpha})=\mathbf{v}^{\prime} \cdot \mathbf{n} /\left|\mathbf{v}^{\prime} \| \mathbf{n}\right|$.

The vector $\mathbf{v}^{\prime}$ is obtained by taking the local down-shaft vector in the club sensor's coordinate system and rotating it by the current orientation of the device. This produces a vector that points down the shaft of the club in the world coordinate system. The club sensor is mounted such that the positive $\mathrm{x}$-axis of the device points down the shaft of the club.

If $\mathbf{q}$ is the quaternion specifying the orientation of the device and $\mathbf{v}_{\text {local }}$ is the vector pointing down the shaft in the local coordinate frame and $\mathbf{v}_{\text {world }}$ is the shaft vector in the world coordinate system, then:

$\mathbf{v}_{\text {world }}=\mathbf{q}^{*} \times \mathbf{v}_{\text {local }} \times \mathbf{q}$, where “*” is the conjugate and " $\mathrm{x}$ " is the quaternion product.

This rule returns $1-\cos (\boldsymbol{\alpha})$ as a score, which is 1 when the swing is perfectly within the plane, and reducing to 0 when perpendicular to the swing plane. 


\section{2 'Head Movement' Rule}

The head movement rule measures the change in orientation of the head about each axis between consecutive snapshots of the golf swing. An Orient- 2 speck is required to be placed on the golfer's cap which acts as the root of the body model and any head movement that is recorded is relative to the motion of the body.

For each snapshot, a world direction vector that points along each axis of the head-mounted device is calculated, using the same method outlined in Section 6.1. This is compared to the previous value to obtain a change in head angle about each axis. The score which is returned by the rule isthen calculated as follows:

if $\delta \mathrm{x}, \delta \mathrm{y}, \delta \mathrm{z}$ are changes in alignment along the $\mathrm{x}, \mathrm{y}$ and $\mathrm{z}$ axis respectively, then the score $=(\operatorname{Abs}(\cos (\delta x))+\operatorname{Abs}(\cos (\delta y))+$ $\operatorname{Abs}(\cos (\delta z))) / 3$.

This formula assumes that the head will not move more than a right angle about any axis between consecutive snapshots of the swing.

The change in angle about each axis is also returned as extra information. This allows the user to see by how much the head had moved about a given axis during the swing.

\section{SOFTWARE}

This section describes the PC and PDA software that interfaces to the sensors and provides motion tracking, analysis and feedback to the user.

\subsection{Data Flow}

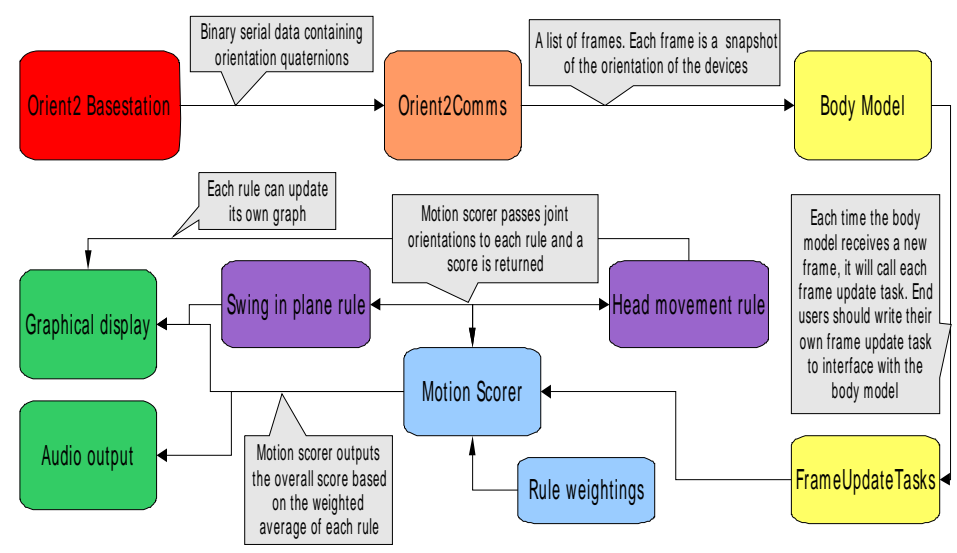

Figure 3: Data flow in the Speckled Golfer System

Figure 3 shows the flow of data from the Orient- 2 specks to the graphical and audio feedback provided to the user. The specks transmit a synchronised snapshot of their orientation to a basestation speck attached to the PC or PDA via a serial connection and provides binary data containing a list of frames. Each frame contains a synchronised orientation quaternion and acceleration vector for each speck on the golfer-club system.

The Orient-2 speck communications module unpacks Frame objects from the binary data and passes them to the Body Model, which maintains a snapshot of the relative orientation of the golfer's joints. The information in the body model is used by many modules for motion visualisation, and analysis, and output to animation packages. Each user of the body model data provides a Frame Update Task, which is run by the body model each time it is updated with new orientation information.

The Motion Scorer's Frame Update Task updates the Motion Scorer module with the latest orientation of the golfer's joints. This is used to obtain a score from each motion rule, as described in Section 6. The scores are weighted, according to a Rule Weightings Table to produce an overall score for the latest snapshot of the swing.

The output from each rule (score and additional information), along with the overall score is passed to the graphical display. The overall score is passed to the Audio Output module which provides real-time feedback of the overall quality of the swing.

\subsection{PC software}

The PC-based software is written entirely in Java. This handles serial communication with the wireless basestation, motion capture, analysis and feedback to the user.

JFreeChart [13] was used to provide the real-time graphs for the golf swing analysis and to show the rule weightings used for the overall score. The graphs can be scaled to show fine details of the motion.

Figure 4 shows the analysis of two golf swings by the Motion Scorer. The first swing is a good one, as it obeys the 'Swinging in a Plane' and the 'Head Movement' motion rules. The second swing deliberately fails to satisfy both the motion rules, by moving well outside the swing plane and the golfer moving his head during the swing.

The overall score graph stays close to 1 for the first swing, but it dips considerably during the second swing. The first dip is caused by the 'Swinging in a Plane' score, which detects the club moving out of the swing plane. The second dip is caused by movement of the head. The auxiliary information displayed by the head movement rule shows that there was significant change in the orientation of the $\mathrm{x}$ axis of the head sensor.

\subsection{PDA software}

A PDA version of the Motion Scorer software enables the Speckled Golfer system to be used in situations where carrying a laptop is inconvenient. This was also written in Java and can run on platforms that support the javacomm serial port communication package and Swing GUI.

The PDA software was tested on an HP rx1950 PDA with a Samsung $300 \mathrm{MHz}$ processor, running Windows Mobile 5. The JVM chosen was the NSIcom CrE-ME 4.12 as this supports J2ME CDC/Personal Profile 1.0, javacomm and swing [14].

The PDA version of the software is fully event-driven, with new data from the base station causes the body model, motion scoring 
system and GUI to be updated. Real-time feedback of the current score produced by each motion rule is provided. The use of multiple threads for Orient-2 data reading, writing and GUI update was found to be extremely slow.

For performance reasons, the full graph of the motion scores over time will be displayed once the motion capture has ended. Full rendering of the 3D body model is not attempted on a lightweight device. Instead, the user can select a single joint of interest to be visualised.

The GUI uses a tabbed interface to switch between the body model selection and sensor-joint mapping, serial communication setup, sensor orientation and motion scoring pages. Due to the small screen size of $320 x 240$ pixels, it was impractical to fit all the information displayed in the PC version onto a single PDA screen.

\section{CONCLUSIONS}

This paper has described the first implementation of a fully wireless inertial sensor network for full-body 3-D motion capture as applied to the analysis of golf swings in real-time which provides a wealth of biomechanical data than hitherto possible. The golfer-club system is modelled as a double pendulum. The factors which affect the efficiency of the golf swing are outlined, such as the length of the back swing, the wrist-cock angle, swing plane and club-head speed and how they can be monitored using the Orient- 2 specks. The rationale for the Motion Scoring System was described and two examples were presented of motion rules for a golf swing: 'Swinging in a Plane' rule and 'Head Movement' rule. The advantages of this approach over current wireless approaches have been described. Future work will involve systematic analysis of the user's experience for a set of golfers.

\section{ACKNOWLEDGEMENTS}

This work was supported in part by the Scottish Funding Council under the Strategic Research Development Grant \#R37329, and the UK Engineering and Physical Sciences Research Council, under the Basic Technology Research Programme Grant \#
C523881. The authors wish to thank the members of the Speckled Computing Group at the University of Edinburgh, and in particular, Alex Young and Martin Ling, for discussions and feedback.

\section{REFERENCES}

[1] Jorgensen, T. P. The Physics of Golf, Springer, 1999.

[2] White, R. On the efficiency of the golf swing, Am. J. Phys., 74, 1088-1094, 2006.

[3] Daish, C. B. The Physics of Ball Games, The English Universities Press, London, ISBN 0340053992, 1972.

[4] Penner, R. “The Physics of Golf”, Rep. Prog. Phys., 66, \#2, 131-171, 2003.

[5] Young, A. D., Ling, M. J., Arvind D. K., “Orient-2: A realtime wireless posture tracking system using local orientation estimation”, in Proc. $4^{\text {th }}$ Workshop on Embedded Networked Sensors, Cork, pp 53-57, June 2007, ACM, 2007.

[6] Arvind, D. K. "Speckled Computing”, in Proc. Nanotech2005, vol 3, pp 351-354, ISBN 0-9767985-2-2, Anaheim CA, USA, May 2005.

[7] Research Consortium in Speckled Computing. http://www.specknet.org

[8] Xsens. http://www.xsens.com

[9] Sonic Golf. http://www.sonicgolf.com

[10] Smart Swing. http://www.smartswinggolf.com

[11] K-Vest. http://www.k-vest.com

[12] Intersense IneriaCube. http://www.isense.com

[13] JFreeChart. http://www.jfree.org/jfreechart

[14] NSIcom CrEME. http://www.nsicom.com 


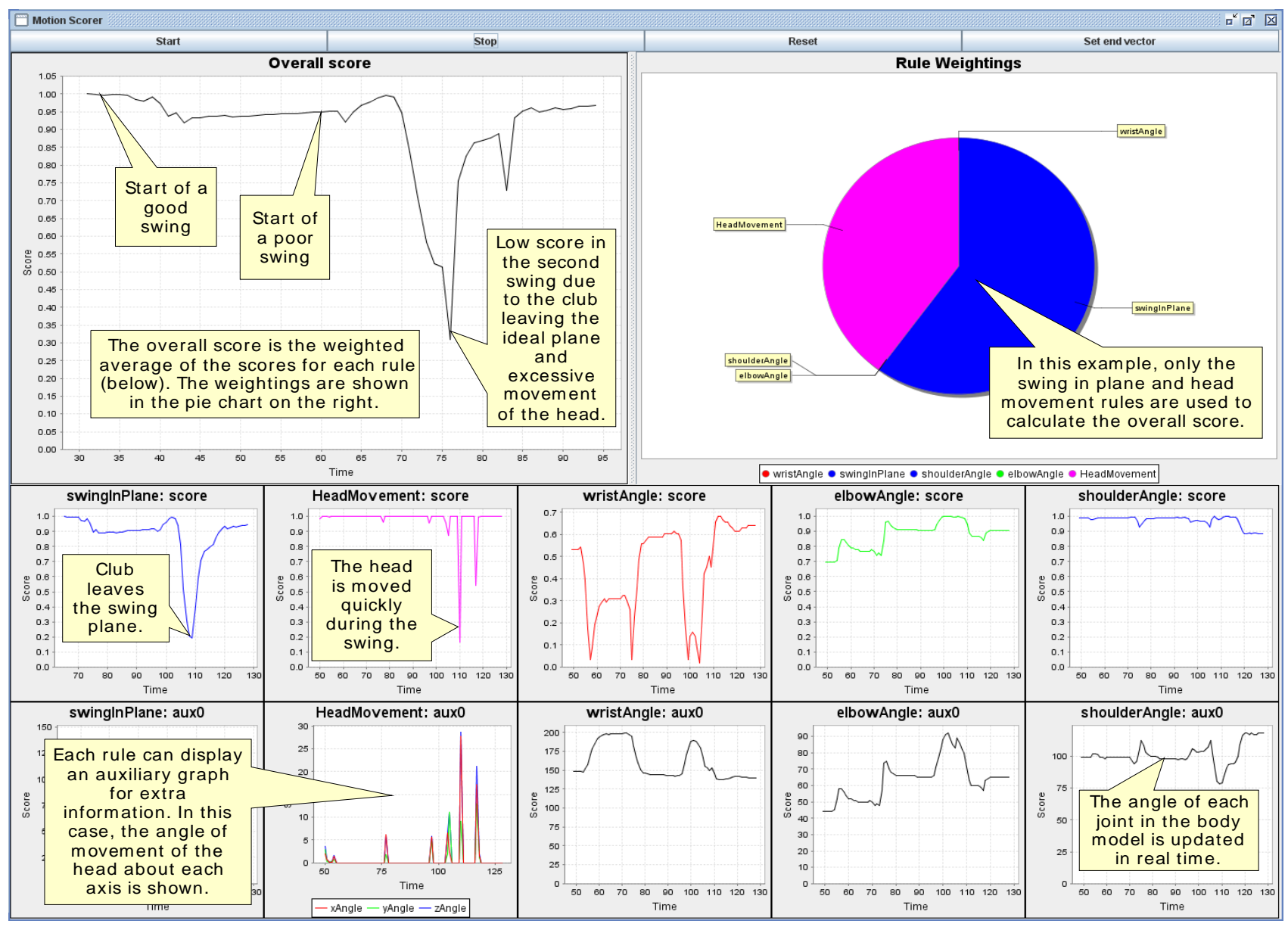

Figure 4: Analysis (with annotations) by the motion scorer for two golf swings 